\title{
FORUM
}

\section{Geringe Hoffnung auf rasche Lösung des Zypern-Konflikts: Kollateralschaden der Finanzkrise?}

\author{
Manuel Sarrazin*
}

Die dramatische finanzielle Rettung Zyperns im ersten Halbjahr 2013 hat die Europäische Union nicht nur vor negativen finanz-, wirtschafts- und währungspolitischen Auswirkungen bewahrt, sondern auch vor einem massiven Verlust an Einfluss in der konfliktreichen Region des östlichen Mittelmeeres. Dennoch hat die Art und Weise der Rettung eine Lösung zweier Schlüsselkonflikte erheblich erschwert und den Einfluss europäischer Akteure auf die Stabilität in der Region geschwächt. Bei den Schlüsselkonflikten handelt es sich um die seit 1974 bestehende Teilung Zyperns und die künftige Ausbeutung der Erdgas- und Ölvorkommen in der Region.

Die innenpolitisch motivierten Debatten im Zuge der ,Zypern-Rettung', vor allem zwischen Teilen der öffentlichen Meinung in Deutschland und Zypern, haben dabei nicht nur Einfluss auf die Ausgestaltung der wirtschaftlichen Lösung der Krise genommen, sondern auch die Chancen zur Lösung des Zypern-Konflikts vermindert. Dabei könnte gerade eine Konfliktlösung wesentlich zum wirtschaftlichen Erfolg des Kreditprogramms beitragen. Gleichzeitig könnte sich in absehbarer Zeit das vielleicht letzte ,Fenster der Gelegenheit` für eine Wiedervereinigung der Insel schließen. Um das zu verhindern, müssen Deutschland, Zypern und die Europäische Union das Krisenmanagement verbessern und ihr Engagement im Friedensprozess stärken.

\section{Die Auswirkungen der wirtschaftlichen Lage auf die Friedensgespräche}

Der Vereinbarung über Kredithilfen für Zypern mit der Troika aus Europäischer Kommission, Internationalem Währungsfonds (IWF) und Europäischer Zentralbank (EZB) gingen einige der dramatischsten Entwicklungen der bisherigen Krise vorweg. Vor allem zwischen den zwei Entscheidungen der Eurogruppe mit dem zwischenzeitlich negativen Votum des zypriotischen Parlaments hatte sich die Lage dramatisch zugespitzt. Für ungefähr eine Woche schien sogar das Überleben des Euro als Währung von 17 beziehungsweise bald 18 EU-Mitgliedstaaten gefährdet und ein Abwenden Zyperns von der europäischen Integration möglich. Die Bürgerinnen und Bürger der Republik Zypern erlebten zeitgleich und innerhalb von nur wenigen Tagen, wie die beiden größten Banken des Landes vor dem Bankrott standen, Kapitalverkehrskontrollen eingeführt und für einen Großteil der Zyprioten der Zugriff auf ihre Bankkonten gesperrt wurde. Gleichzeitig ereilte Zypern ein ökonomischer Schock: Der IWF rechnet für die Jahre 2013 und 2014 mit einem Einbruch der Wirtschaftsleistung Zyperns um insgesamt 13 Prozent des Bruttoinlandsprodukts (BIP). ${ }^{1}$ Schon 2012 ist die Wirtschaft, die sich 2010 und 2011 zunächst leicht von der Rezession im Zuge der weltweiten

\footnotetext{
* Manuel Sarrazin, MdB, Sprecher für Europapolitik und Obmann im Europaausschuss, Fraktion Bündnis 90/Die Grünen.

1 Vgl. EU-Info.Deutschland: IWF: Zyperns Wirtschaft dürfte um 13 Prozent schrumpfen, 1.8.2013.
} 
Finanzkrise 2009 erholt hatte, um 2,4 Prozent des BIP geschrumpft. ${ }^{2}$ Die Arbeitslosigkeit verzeichnete den EU-weit stärksten Anstieg und belief sich - saisonbereinigt - im Juni 2013 auf ein Rekordhoch von 17,3 Prozent, Tendenz weiter steigend. ${ }^{3}$

Zeitgleich sollen die unter dem Dach der Vereinten Nationen (VN) angesiedelten Gespräche über eine mögliche Lösung des Zypernkonflikts im Oktober 2013 wieder aufgenommen werden. ${ }^{4}$ Diese Meldungen haben nur auf den ersten Blick nichts miteinander zu tun. Tatsächlich aber haben die Umstände und politischen Begleitdebatten der ,Zypern-Rettung' die Chancen auf eine Annäherung in den Verhandlungen zur Lösung des ZypernKonflikts erschwert.

Seit der Teilung im Jahr 1974 in Folge der Invasion türkischer Truppen im Norden Zyperns gab es mehrfach Versuche, die Insel wieder zu vereinigen. Doch allein in den letzten 15 Jahren sind zwei Versuche in fortgeschrittenem Stadium gescheitert, der letzte im Jahr 2004, als der sogenannte Annan-Plan zur Wiedervereinigung in einem Referendum von 76 Prozent der griechisch-zypriotischen Bevölkerung abgelehnt wurde. Neue Hoffnung auf eine Lösung verband sich mit der Wahl des neuen zypriotischen Präsidenten Nicos Anastasiades im Februar 2013. Anastasiades, der als damaliger Befürworter des Annan-Plans politische Glaubwürdigkeit für eine Lösung mitbringt, hatte schon im Wahlkampf angekündigt, die Verhandlungen wiederbeleben zu wollen. ${ }^{5}$

\section{Die Zypernrettung und der Zypernkonflikt: die Gemengelage}

Dieses vielversprechende Momentum ist im Zuge der ,Zypern-Rettung ' zumindest vorerst verspielt. Eine seit November 2012 in deutschen und europäischen Medien sowie teilweise in der Politik undifferenziert und überdimensioniert geführte Debatte über wettbewerbsverzerrende Niedrigsteuern, russische Oligarchen und Geldwäsche auf Zypern, hatte in der zypriotischen Öffentlichkeit schnell eine ,nationale Abwehrreaktion` zur Folge. Zypern wurde dort zum Opfer einer vor allem von Berlin gesteuerten europäischen Politik stilisiert. Die Frage der finanziellen Rettung Zyperns, die ohnehin mit sehr unterschiedlichen Interessen und schwierigen Entscheidungen sowohl für Zypern als auch für die europäischen und internationalen Partner verbunden war, geriet zu einer Frage des nationalen Prestiges, anstatt die hinter den Meinungsverschiedenheiten liegenden Interessenunterschiede herauszuarbeiten und die notwendigen Kompromisslinien in der Kommunikation vorzuzeichnen.

Auch die Debatte in Deutschland erweckte teilweise den Eindruck, als ob es eigentlich nur um die Rettung von russischem Schwarzgeld, Geldwäschekapital und Steuerhinterziehern gehe. ${ }^{6}$ Die existenziellen Ängste und Sorgen der Menschen auf Zypern wurden ebenso wie die besondere Situation der geteilten Insel weitgehend ignoriert. Weder die Bundesregierung noch die deutsche Opposition haben in dieser Situation korrigierenden Einfluss auf die Debatte in Deutschland genommen. Die Schlagzeilen in Deutschland haben in Zypern einen tiefen Eindruck hinterlassen, oftmals mehr als im Bewusstsein der Deutschen selbst.

2 Vgl. Auswärtiges Amt: Wirtschaft Zypern, Juni 2013, abrufbar unter: http://www.auswaertiges-amt.de/sid_E83 8EB612564716A0D8D1A75812BC56A/DE/Aussenpolitik/Laender/Laenderinfos/Zypern/Wirtschaft_node.htm 1\#doc358800bodyText2 (letzter Zugriff: 21.10.2013).

3 Vgl. Europäische Kommission, Generaldirektion Wirtschaft und Finanzen: Das wirtschaftliche Anpassungsprogramm für Zypern, Erste Prüfung - Sommer 2013, Entwurf, August 2013, S. 11.

4 Vgl. EU-Info.Deutschland: Zypern-Gespräche sollen im Oktober weitergehen, 30.7.2013.

5 Vgl. Hubert Faustmann: Öl- und Gas vor Zypern. Entsteht ein neuer Krisenherd im östlichen Mittelmeer?, in: Wissenschaft \& Frieden 2/2012, S. 19-22.

6 Vgl. SpiegelOnline: Russische Schwarzgeldkonten: BND warnt vor Rettungspaket für Zypern, 3.11.2012. 
Sie haben in Zypern bei den Bürgerinnen und Bürgern zu Wut, Ablehnung und einem erheblichen Akzeptanzverlust der europäischen Integration beigetragen. Gleichzeitig konnten dadurch die tatsächlichen, hausgemachten Probleme Zyperns durch eine Debatte der ,nationalen Verletztheit' überlagert werden. Anstatt die Verantwortung derjenigen zu thematisieren, die mitten in der Griechenlandkrise das finanzielle Engagement der zypriotischen Banken in Griechenland auch noch vergrößerten, bot der, Antagonismus' zu Berlin Vielen eine bequeme Ausflucht. Für Deutschland, aber auch für Zypern galt: In Teilen der Medien und der Politik wurde es plötzlich zum Allgemeingut, populistisch und despektierlich übereinander zu reden; ein einseitiges, undifferenziertes Bild vom jeweils anderen zu erzeugen und die existenziellen Sorgen der Menschen auf Zypern, aber auch die berechtigte Kritik an der Finanzpolitik auf Zypern nur unzureichend zu erwähnen. Die Debatte machte die ,Rettung ‘ - unabhängig von der materiellen Bewertung des vereinbarten Kreditpakets - aus zypriotischer Sicht letztlich nicht allein wegen seiner wirtschaftlichen Folgen zu einer nationalen Niederlage, sondern schmälerte auch das politische Kapital des frisch gewählten Präsidenten. Verschlimmert wurde diese Lage zudem dadurch, dass bis zur letzten Minute die Kommunikation aus Deutschland zwar auf die Notwendigkeit eines Umbaus des sogenannten Geschäftsmodells Zyperns einging, dabei aber der Weg aus der Krise als alleinige Aufgabe Zyperns beschrieben wurde. Nicht einmal in der Rhetorik wurden in der deutschen Politik die wirtschaftlichen Lasten der Bürgerinnen und Bürger in Zypern ausreichend gewürdigt und die eigene Bereitschaft wie auch die Notwendigkeit zu europäischer Solidarität hervorgehoben. ${ }^{7}$

\section{Die wirtschaftlichen Folgen der Krise für Zypern}

Die wirtschaftlichen und politischen Folgen der Krise sind außergewöhnlich hart. Um die entscheidende Maßgabe der Schuldentragfähigkeit zu erreichen, muss Zypern seinen Staatshaushalt durch Ausgabenkürzungen und eine Verbesserung der Einnahmenseite insgesamt um 2 Prozent des BIP konsolidieren. ${ }^{8}$ Im Zuge der jahrelang expansiven Politik des Finanzplatzes Zypern, dessen Gesamtvolumen zuletzt mehr als das Siebenfache der eigenen Wirtschaftsleistung betrug, der Griechenlandkrise und des griechischen Schuldenschnitts häuften sich die Probleme in den Banken Zyperns, die allerdings kaum international vernetzt sind. ${ }^{9}$ Zur Finanzierung der Restrukturierung muss Zypern weitgehend auf die Einlagenbeträge über 100.000 Euro bei den beiden größten Banken des Landes zugreifen. ${ }^{10}$ Der Umbau der größten Bank des Landes, der Bank of Cyprus (BoC), gestaltet sich schwierig. Ohne eine erfolgreiche Restrukturierung und Wiederherstellung des Liquiditätszugangs der BoC, wird Zypern jedoch die Kapitalverkehrskontrollen in absehbarer Zeit nicht aufheben können. Nach wie vor lähmen diese das Land wirtschaftlich und führen zu unzähligen Konkursen unter den kleinen und mittleren Unternehmen. Unter der mit der Bankenkrise einhergehenden Rezession, die unter anderem durch den Zusammenbruch des Bausektors und des Geldverkehrs verursacht wurde, fällt es vielen Menschen und Unternehmen schwer, ihre Gehälter und Rechnungen einzutreiben beziehungsweise zu bezahlen und folgelogisch auch ihre Kre-

7 Vgl. Faz.net: Schäuble: Zypern muss sein Geschäftsmodell ändern, 20.3.2013.

8 Vgl. Europäische Kommission: Bewertung der Tragfähigkeit der Staatsverschuldung Zyperns, Arbeitsübersetzung des Bundesministeriums der Finanzen, 12.4.2013, S. 7.

9 Vgl. Deutscher Bundestag: Sachstandsbericht des Referates EU-Grundsatzangelegenheiten und Fragen der Wirtschafts- und Währungsunion im Bundestag: Die Programmländer Griechenland, Irland, Portugal, Spanien sowie Zypern: Ein Überblick, S. 14.

10 Vgl. Eurogruppe: Eurogroup-Statement on Cyprus, 25.3.2013. 
dite zu bedienen. Das vergrößert wiederum die Probleme bei der Restrukturierung der Banken.

\section{Die Zusammenarbeit zwischen Norden, Süden und der Türkei als Chance für wirtschaftliche Wiederbelebung}

In dieser extrem schwierigen Situation könnten Fortschritte im Friedensprozess zum Beispiel in den Bereichen bizonaler Zusammenarbeit, Handel mit der Türkei oder die Lösung der Famagusta-Frage ${ }^{11}$ konkrete wirtschaftliche Impulse bringen. Diese Fortschritte könnten in Zypern Wachstum generieren, attraktive Investitionsmöglichkeiten schaffen und somit der befürchteten weiteren massiven Kapitalflucht entgegentreten. Gleichzeitig würde auch der Norden der Insel wirtschaftlich profitieren - ein Umstand der vor dem Hintergrund der ebenfalls harten Verhandlungen über die finanziellen Hilfen der Türkei an den türkischzypriotischen Norden interessant wäre. Fortschritte im Friedensprozess würden also allen Seiten wirtschaftliche Vorteile bieten und zu einem Erfolg des Kreditprogramms beitragen.

\section{Die Europäische Union muss zusätzliche Unterstützung anbieten}

Unabhängig davon ist natürlich die Europäische Union gefragt, der Bevölkerung Zyperns jedwede mögliche Unterstützung zukommen zu lassen, damit Zypern schnellstmöglich wieder wirtschaftlich gesundet. Dazu gehören in erster Linie eine rasche und entschiedene Strategie für die Restrukturierung der BoC, eine schnelle und unbürokratische Nutzung von Mitteln aus den Regional- und Strukturfonds, die Unterstützung bei der Mittelstandsfinanzierung, zum Beispiel durch eine Mittelstandsförderbank und die Verwaltungsunterstützung durch eine Task-Force der Europäischen Kommission sowie durch bilaterale Projekte mit EU-Partnern.

\section{Der Friedensprozess ist in Gefahr}

Die wirtschaftliche Lage auf der Insel hat in Verbindung mit der unglückseeligen Debatte über verletzten nationalen Stolz in Teilen der Öffentlichkeit Zyperns zu einer Situation geführt, in der sich die Hoffnungen auf rasche Fortschritte im Friedensprozess nicht realisiert haben. Vielmehr ist für die griechischen Zyprioten erste Priorität, die weiterhin das Land bedrohenden finanz- und wirtschaftspolitischen Probleme zu lösen, bevor mit einem ernsthaften Fortgang der Verhandlungen zu rechnen ist. Sollten Zypern und die Eurozone daran scheitern, eine tragfähige Lösung für die BoC und die Aufhebung der Kapitalverkehrskontrollen zu finden, wird dies voraussichtlich auch die noch im Jahr 2013 beginnenden, von den Vereinten Nationen geführten Friedensgespräche zwischen dem türkisch-zypriotischen Norden und dem griechisch-zypriotischen Süden überschatten. Es scheint unrealistisch, dass ohne eine Stabilisierung der ökonomischen Lage die Entscheidungsträger im Süden der Insel die Voraussetzung für die Durchsetzbarkeit von relevanten Fortschritten im Friedensprozess für gegeben halten.

Gleichzeitig könnten die Präsidentschaftswahlen in der Türkei im August 2014 und die Präsidentschaftswahlen im Norden der Insel 2015 das Zeitfenster für rasche Lösungen nach hinten begrenzen. Vor dem Hintergrund der steigenden Abhängigkeit des Nordens von An-

11 Famagusta ist eine Hafenstadt an der Ostküste von, Nordzypern‘. Territoriale Konflikte, vor allem um den Vorort Varosha, sind ein wesentlicher Streitpunkt weiterer Friedensverhandlungen. 
kara, der strukturellen Veränderungen in der Gesellschaft Nordzyperns durch den Zuzug von Siedlern vom türkischen Festland, der im Süden der Insel anstehenden Präsidentschaftswahlen 2017 und der Weiterentwicklung der EU-Beitrittsverhandlungen mit der Türkei ist zudem davon auszugehen, dass die Einigungschancen mit fortschreitender Zeit kleiner werden. Manch ein Freund der Insel spricht schon heute vom vielleicht letzten Zeitfenster für eine Lösung des Zypern-Konflikts.

\section{Die Rolle der Gasvorkommen ${ }^{12}$}

Auch die Strategie der südzypriotischen Regierung zur Ausbeutung, Verarbeitung und Vermarktung der vermuteten Gasvorkommen in der ausschließlichen Wirtschaftszone der Insel wird durch den fehlenden Fortschritt der Zypern-Verhandlungen beeinflusst. Auch hier scheinen die unterschiedlichen Positionen verhärtet. Obwohl die Ergebnisse erster Erkundungsbohrungen im sogenannten Block 12 hinter den erwarteten Erdgasmengen zurückblieben, scheint die zypriotische Regierung nicht von ihrer Grundsatzentscheidung über den Bau eines Flüssiggas-Terminals (LNG-Terminals ${ }^{13}$ ) abzurücken. Diese wird den Süden zumindest vorläufig auf eine Gasstrategie festlegen, die den Export unabhängig von der Türkei per Schiff und damit ohne ein türkisches Faustpfand für ernsthafte Verhandlungen über die Nutzung der Gasvorkommen in Kooperation mit dem Norden der Insel entwickeln will. Die zwischenzeitlich geplante Beteiligung der Bevölkerung Nordzyperns an den Erlösen des Rohstoffgeschäfts wird in der derzeitigen wirtschaftlichen Lage auch politisch hinter dem Argument des wirtschaftlichen Überlebens der ,Nation' zurücktreten. ${ }^{14}$ Damit wird das Konfliktpotenzial in der Ressourcenfrage - die in den von den Vereinten Nationen geführten Zypern-Gesprächen ausgeklammert werden soll - zumindest vorläufig eher zunehmen. ${ }^{15} \mathrm{Zu}$ befürchten ist, dass sich zunehmende Spannungen in der Energiefrage negativ auf diese Verhandlungen auswirken könnten, da Nordzypern und die Türkei auf eine Beteiligung an den Erdgasgewinnen bestehen werden. Die Hoffnung mancher Beobachter, dass die Erdgasfunde wirtschaftliche Anreize zur Lösung der politischen Konflikte auf der Insel und in der Region bieten, steht zumindest in den nächsten Monaten dem Interesse der zypriotischen Regierung entgegen, in diesem Bereich schnell Investitionen auf die Insel zu holen und Wachstum zu generieren. Die Investitionen der Erdgasindustrie werden beispielsweise im Bereich Infrastruktur schon jetzt anlaufen, auch wenn mit Erträgen aus dem Erdgas- (und Öl-)Geschäft frühestens am Ende dieses Jahrzehnts zu rechnen ist. Eine Veränderung dieser Gemengelage könnte wohl nur durch eine weitere Verschiebung von Variablen im Bereich Geologie (tatsächliches Volumen der Funde) oder Geopolitik (Realisierung einer Zusammenarbeit mit Israel beim Gasexport über das LNG-Terminal) oder durch Fortschritte bei den Friedensverhandlungen eintreten, welche zu einem Konsens über die Integration der Erdgasthematik führen würden.

12 Die Royal Bank of Scotland hat den Wert der vermuteten Erdgasreserven vor der Südküste Zyperns auf mehr als 600 Milliarden Euro geschätzt. Vgl. Natural Gas Europe: Cyprus Natural Gas Sector Sails on Fast, 4.2.2013, abrufbar unter: http://www.naturalgaseurope.com/cyprus-natural-gas-sector-sails-on-fast (letzter Zugriff: 21.10.2013).

13 ,Liquefied natural gas'.

14 Vgl. United Nations: General Assembly. Sixty-sixth session. $15^{\text {th }}$ plenary meeting. Thursday, 22 September 2011, 11 a.m., New York, A/66/PV.15, S. 1-4.

15 Vgl. Faustmann: Öl und Gas vor Zypern, 2012. 


\section{Das missglückte Krisenmanagement}

Die existenzielle Krise Zyperns, an deren Ausgangspunkt die hier beschriebenen negativen Entwicklungen standen, hat ihre Ursachen in erster Linie im aufgeblähten Finanzsektor der Insel und dem Agieren der Finanzinstitutionen des Landes vor und auch noch während der Griechenland-Krise. Das Geschäftsmodell der Insel musste sich als nicht mehr tragfähig herausstellen. Auf der Insel wurde genauso wenig rechtzeitig entgegengesteuert, wie die Europäische Union in der Lage war, Politikveränderungen zu erwirken oder die Lage des Landes bei der Vereinbarung des Schuldenschnitts für griechische Staatsanleihen mit zu berücksichtigen. Der Schuldenschnitt für Griechenland, der dramatische Wirtschaftseinbruch in Griechenland, die Explosion des Stromkraftwerks in der Nähe von Limasol und die allgemeine Unsicherheit über die Zukunft des Euro hatten die Lage in Zypern bis zum Sommer 2012 entscheidend zugespitzt. ${ }^{16}$ Ausgerechnet in dieser dramatischen Situation war die zypriotische Regierung nicht in der Lage, die Verhandlungen mit den europäischen und internationalen Geldgebern entscheidend voranzutreiben und so die Lage der Insel zu stabilisieren. Gleichzeitig bewahrheitete sich, dass von dem erforderlichen Finanzvolumen zur Stabilisierung der zypriotischen Banken nur ungefähr 10 Milliarden Euro von den internationalen Geldgebern gestellt werden würden. ${ }^{17}$ Offenkundig unterschätzte die zypriotische Politik bis zuletzt den sehr engen Bewegungsspielraum ihrer Partner: Es gab bei IWF und EZB massive Auseinandersetzungen über die Gestaltung ihres Engagements in Griechenland und in Zypern. Im Hintergrund verhandelten die Euro-Finanzminister über die grundsätzlichen Regeln der künftigen europäischen Bankenunion und in der deutschen Bundesregierung wollte man zudem einen Präzedenzfall verhindern, der die gerade vereinbarten Prinzipien und Regeln des Europäischen Stabilitätsmechanismus (ESM) faktisch außer Kraft gesetzt hätte. Infolgedessen war keiner der Geldgeber bereit, die Schuldenlast Zyperns über einer Marge von rund 100 Prozent des BIP zum Ende des Jahrzehnts als tragfähig anzusehen und Hilfen in Höhe des kompletten Finanzbedarfs von rund 17 Milliarden Euro bereitzustellen. Nachdem sich die Möglichkeit zur Finanzierung des Fehlbetrags durch externe Partner wie Russland oder China zerschlagen hatte, scheiterte ein erster Versuch Zyperns und der Eurogruppe, diese Lücke mit einer Beteiligung (auch unter 100.000 Euro) beziehungsweise einer Besteuerung von Bankeinlagen auf Zypern zu schließen. Eine solche Entscheidung wäre in keiner Weise - weder auf Zypern noch in der Eurozone insgesamt - zu legitimieren gewesen, da sie das Vertrauen in die europäische Einlagensicherung schwer erschüttert und Verwerfungen an den Finanzmärkten in ganz Europa ausgelöst hätte.

Nach der Ablehnung dieser Entscheidung durch das zypriotische Parlament führten dramatische Verhandlungen in letzter Minute zu einer Lösung im Rahmen einer zweiten Entscheidung der Eurogruppe: Alle Gläubiger und Anleger von mehr als 100.000 Euro der beiden größten Banken des Landes wurden umfassend an den Verlusten beteiligt. ${ }^{18}$ Diese

16 Vgl. Gert Höhler: Explosion macht Zypern zum vierten Hilfskandidaten, in Handelsblatt, 13.7.2011: „Die zyprische Europa-Abgeordnete Antigoni Papadopoulou bezifferte die Schäden der Explosion auf rund 3,5 Milliarden Euro. Das entspräche 20 Prozent des Bruttoinlandsprodukts (BIP) der Insel oder 43 Prozent des Staatshaushalts“; „Nach einer Schätzung der Agentur Standard \& Poor's halten zyprische Banken griechische Staatsund Bankanleihen im Nennwert von 6,4 Milliarden Euro. Das entspräche etwa 37 Prozent des BIP Zyperns“.

17 Die Eurogruppe hat das Programm mit einem Finanzvolumen von 10,0 Milliarden Euro am 8. Mai 2013 beschlossen, hiervon tragen der Europäische Stabilitätsmechanismus 9,0 Milliarden Euro und der Internationale Währungsfonds 1,0 Milliarden Euro. Vgl. Bundesministerium der Finanzen: Ausführungen zur aktuellen Inanspruchnahme des Europäischen Stabilitätsmechanismus (ESM), Juni 2013.

18 Vgl. Eurogruppe: Eurogroup-Statement on Cyprus, 25.3.2013. 
Lösung, die auf Zypern zu stärkeren wirtschaftlichen Verwerfungen im Vergleich zur ersten Entscheidung der Eurogruppe führte, stellte das Vertrauen in die Einlagensicherungssysteme der Eurozone insgesamt wieder her und beruhigte so die heikle Lage für den Rest der Eurozone, während Zypern die kurzfristig eingeführten Kapitalverkehrskontrollen nicht wie vorgesehen innerhalb weniger Tage wieder aufheben konnte. Ausgehend von auf Zypern selbst liegenden Ursachen eines nicht ausreichend regulierten und aufgeblähten Finanzsektors, führten fehlende und falsche politische Entscheidungen sowie mangelnder politischer Spielraum aller entscheidenden Akteure zu großen wirtschaftlichen und politischen Verwerfungen für Zypern. Ein unkontrollierter Bankrott der Republik Zypern hätte allerdings mit Sicherheit noch viel drastischere und gefährlichere wirtschaftliche und politische Folgen erzeugt.

\section{Worauf es ankommt: europäischer Zusammenhalt}

In den öffentlichen Debatten im Zuge der politischen ,Rettungsentscheidungen“ werden zumeist vor allem wirtschaftliche und finanzpolitische Argumente angeführt, um die Situation in den kreditnehmenden Staaten und die dort notwendige Transformation zu beschreiben. Das Beispiel Zypern zeigt, wie die in den Medien und auch in der Politik geführte innenpolitisch motivierte öffentliche Debatte in Deutschland Lösungen erschweren oder zu politischen ,Kollateralschäden“ beitragen kann, sofern die Politik nicht in der Lage ist, ein realistisches und faires Bild der europäischen Partner zu präsentieren und den Menschen ein Verständnis für die Probleme in den sogenannten Krisenstaaten zu vermitteln. Es liegt im deutschen Interesse zu einer erfolgreichen Transformation von kreditnehmenden Staaten beizutragen. Dafür muss die - demokratiepolitisch unerlässliche - öffentliche Debatte in Deutschland sich der Wirkung der eigenen Aussagen auf die öffentliche Meinung in den europäischen Partnerländern bewusst sein und es muss über sogenannte Krisen-Länder sowie anstehende Entscheidungen auf einem ausreichend fachlichen Niveau politisch diskutiert werden. Es ist zuallererst Aufgabe der Regierung, dementsprechend zu informieren und zu kommunizieren. Aber auch die Opposition muss parteipolitische und persönliche Interessen in diesen Situationen zurückstellen zugunsten des Wertes des Zusammenhalts in Europa.

Das Beispiel Zypern zeigt: Die argumentative Vormacht in der öffentlichen Debatte darf nicht denjenigen überlassen werden, die in der Krise zunehmend Bilder der gegenseitigen ,Verletzung beziehungsweise Verletztheit nationaler Gefühle‘ erzeugen und die Krise immer mehr durch eine ,Sündenbocklogik‘ zu erklären suchen. Künftige Debatten werden zeigen, ob Deutschland seiner neuen Führungsrolle in Europa dann besser gerecht werden kann, als es die deutsche Debatte im Fall Zypern getan hat. 